Full Paper

\title{
Electrochemical Impedance Characterization of Nafion-Coated Carbon Film Resistor Electrodes for Electroanalysis
}

\author{
Carla Gouveia-Caridade, Christopher M. A. Brett* \\ Departamento de Química, Universidade de Coimbra, 3004-535 Coimbra, Portugal \\ *e-mail:brett@ci.uc.pt
}

Received: May 3, 2004

Final version: July 8, 2004

\begin{abstract}
Carbon film disk electrodes with Nafion coatings have been characterized by electrochemical impedance spectroscopy (EIS) with a view to a better understanding of their advantages and limitations in electroanalysis, particularly in anodic stripping voltammetry of metal ions. After initial examination by cyclic voltammetry, spectra were recorded over the full potential range in acetate buffer solution at the bare electrodes, electrodes electrochemically pretreated in acid solution, and Nafion-coated pretreated electrodes in the presence and absence of dissolved oxygen. EIS equivalent circuit analysis clearly demonstrated the changes between these electrode assemblies. In order to simulate anodic stripping voltammetry conditions, spectra were also obtained in the presence of cadmium and lead ions in solution at Nafion-coated electrodes, both after metal ion deposition and following re-oxidation. Permanent changes to the structure of the Nafion film occurred, which has implications for use of these electrode assemblies in anodic stripping voltammetry at relatively high trace metal ion concentrations.
\end{abstract}

Keywords: Carbon film resistor electrodes, Nafion polymer, Electrochemical impedance spectroscopy, Anodic stripping voltammetry

\section{Introduction}

The great concern in recent years regarding the toxicity of mercury has contributed to the motivation for developing new electrode materials without mercury for electroanalytical applications. Such materials include various forms of carbon, widely applied as electrodes and as electrode substrates [1]. Glassy carbon, in particular, has played an important role in voltammetric studies due to its low permeability to gases, low porosity, hardness, good electrical conductivity and a good positive potential range.

Nevertheless, the surfaces of solid electrodes, such as glassy carbon, tend to become blocked over time when employed in analyzes in natural media due to irreversible adsorption of electroactive and other chemical species, leading to a decrease in response and difficulties in the analysis of untreated samples [2]. Modifying or protecting the electrode surface can lead to minimization of such factors and to a useful electrode material for electroanalysis.

Polymer modification has been used with success for reducing adsorption phenomena and for protecting electrodes. Mercury thin film electrodes on glassy carbon electrode substrates with Nafion coatings were prepared and applied to determination of metal ions by batch injection analysis (BIA), a technique in which sample volumes are of the order of $50 \mu \mathrm{L}$ and can be injected into electrolyte without any sample pretreatment [3]. The Nafion coating reduces the signal by about $15 \%$ but confers physical stability to the mercury film and some extra selectivity due to its cationexchange properties. Electrochemical impedance spectro- scopy (EIS) was shown to be a powerful tool for the characterization of such Nafion-coated mercury thin film electrodes [4]. Mixtures of Nafion and other polymers [5] as well as poly(ester sulphonic acid) cation-exchange coatings have also been investigated for this purpose [6]. Nevertheless, it was concluded that for application to natural media, Nafion coatings, which are the easiest to prepare of those tested, represent sufficiently good discrimination against potential adsorbents and interfering species.

The application of EIS to electrochemical sensor characterization has been relatively infrequent but is increasing, e.g., [7-10], although applications to polymer-modified electrodes have mainly involved conducting-polymers $[7$, 10].

Even though the use of polymer-protected mercury thin films reduces the amount of mercury used and prevents it from being released to the local environment, complete removal is preferable. Carbon film electrodes have been shown to be a promising alternative and several types of carbon film electrode have been investigated with this aim in mind, e.g., [11-14].

In previous publications $[14,15]$ carbon film cylinder electrodes made from electrical resistors have been investigated electrochemically and their application in trace metal analysis by stripping voltammetry demonstrated. Characterization by electrochemical impedance spectroscopy [16] was carried out in several electrolytes used in electroanalytical experiments over a wide range of $\mathrm{pH}$ values, studying the influence of pretreatment of the electrode surface by potential cycling in perchloric acid on 
the voltammetric profile. It was concluded that the reactivity of the electrode surface depends on the electrolyte and on the pretreatment, altering the shape of the impedance spectra and the magnitude of the impedance. Additionally, pretreatment in perchloric acid leads to a more reactive surface, more useful for electroanalytical applications.

The purpose of this work was to characterize Nafioncoated carbon film resistor disk electrodes as used in voltammetric trace analysis by EIS, after examination of the potential window by cyclic voltammetry. Comparison is made with results obtained at Nafion-coated mercury thin film glassy carbon electrodes [4] to investigate the benefits of the mercury-free system and the changes that occur.

The study included electrodes without Nafion before and after pretreatment by potential cycling in acid and in the absence and presence of dissolved oxygen.

\section{Experimental}

\subsection{Electrode Preparation}

\subsubsection{Carbon Film Disk Electrodes}

Electrodes were made from carbon film resistors of $2 \Omega$ nominal resistance: the resistors were fabricated from ceramic cylinders of length $0.4 \mathrm{~cm}$ and external diameter $0.15 \mathrm{~cm}$ by pyrolytic deposition of carbon from methane in a nitrogen atmosphere [14]. One of the two tight-fitting metal caps, linked to wires as external contact, was removed and the other was protected by normal epoxy resin, with the contacting wire being sheathed in plastic. The cylinder was then covered in epoxy resin except on the bottom surface. The final result was a disk electrode of area ca. $0.020 \mathrm{~cm}^{2}$.

Electrochemical pretreatment of a carbon film electrode involved cycling it 3 times in $1.0 \mathrm{M}$ perchloric acid solution between potential limits defined by a maximum current of $\pm 0.1 \mathrm{~mA}$ (approximately between -2.0 and $+2.0 \mathrm{~V}$ vs SCE) at a scan rate of $100 \mathrm{mV} \mathrm{s}^{-1}$ starting at $0.0 \mathrm{~V}$ (vs. SCE) in the positive direction. This pretreatment has been used in other situations [14-16] and improves the width of the potential window.

\subsubsection{Nafion-Coated Carbon Film Disk Electrodes}

A volume of $1 \mu \mathrm{L}$ of $0.25 \mathrm{wt} \%$ Nafion solution was applied to the electrochemically pretreated electrode surface followed by $1 \mu \mathrm{L}$ of $N, N^{\prime}$-dimethylformamide, using an electronic micropipette (EDP-Plus, Rainin). The polymeric film was then cured with a jet of warm air for about $1 \mathrm{~min}$. This Nafion coating procedure leads to a film of ca. $1 \mu \mathrm{m}$ thickness.

\subsection{Instrumentation}

Measurements were made in a three-electrode one-compartment cell containing the working electrode, a platinum foil auxiliary electrode and a saturated calomel electrode (SCE) as reference.

Cyclic voltammetry experiments were carried out using a $\mu$ Autolab potentiostat (Eco Chemie, Utrecht, Netherlands) controlled by GPES 4.9 software.

Electrochemical impedance spectra were recorded using a Solartron 1250 Frequency Response Analyzer coupled to a Solartron 1286 Electrochemical Interface (Solartron Analytical, UK), controlled by ZPlot software. The frequency range from $65.5 \mathrm{kHz}$ to $0.1 \mathrm{~Hz}$ was scanned logarithmically with an applied sinusoidal perturbation of $10 \mathrm{mV}$ rms amplitude in 10 steps per frequency decade, superimposed on the chosen applied potential. Data fitting to equivalent circuits was performed with ZView software.

\subsection{Reagents and Solutions}

All solutions were prepared from analytical grade reagents and Millipore Milli-Q ultrapure water (resistivity $\geq 18 \mathrm{M} \Omega$ $\mathrm{cm}$ ). Unless specified, the supporting electrolyte was $0.1 \mathrm{M}$ sodium acetate/acetic acid buffer ( $\mathrm{pH} 4.4)$. For electrode pretreatment, the solution used was $1.0 \mathrm{M}$ perchloric acid solution and all experiments were carried out at room temperature $\left(25 \pm 1^{\circ} \mathrm{C}\right)$.

To obtain deaerated solutions, ultrapure nitrogen was bubbled through the solution for $10 \mathrm{~min}$ prior to recording impedance spectra.

\section{Results and Discussion}

\subsection{Cyclic Voltammetry}

Before characterization by EIS, the behavior of uncoated and coated carbon film disk electrodes was examined by cyclic voltammetry before and after surface pretreatment and with Nafion coatings, with respect to potential window and background currents.

Cyclic voltammograms (CV) recorded in acetate buffer solution before and after pretreatment by cycling the electrode 3 times in $1.0 \mathrm{M}$ perchloric acid solution between $\pm 0.1 \mathrm{~mA}$, gave similar results to those in [12] at carbon film cylinder electrodes. The potential window increased and the background currents became lower. The positive potential limit increased from $1.8 \mathrm{~V}$ for electrodes before pretreatment to $1.9 \mathrm{~V}$ (vs.SCE) after pretreatment. A more significant change occurred in the negative limit which varied from ca. $-1.8 \mathrm{~V}$ to ca. $-2.0 \mathrm{~V}$ (vs. SCE) after cycling in perchloric acid. Figure 1 shows a typical $\mathrm{CV}$ obtained in acetate buffer solution after pretreatment of the electrode and after coating with Nafion. The coating did not influence the potential window and the effect on the background current was small.

Similar experiments were carried out in $0.1 \mathrm{M} \mathrm{KNO}_{3} /$ $5 \mathrm{mM} \mathrm{HNO}_{3}$ electrolyte solution, also utilized in stripping voltammetric analysis [4]. However, the background current remained high even after electrode pretreatment, with the 


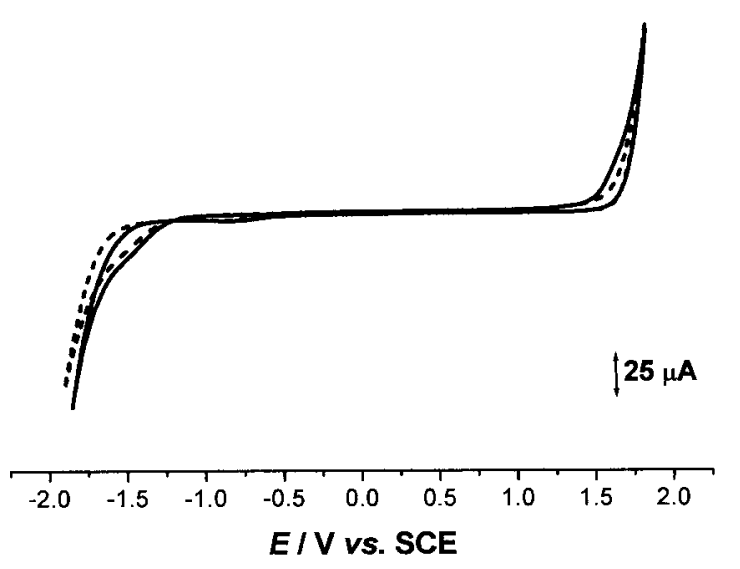

Fig. 1. Cyclic voltammograms in $\mathrm{pH} 4.4$ acetate buffer solution at carbon film disk electrodes, area $0.02 \mathrm{~cm}^{2},(-)$ after pretreatment and (......) Nafion-coated. First cycle, scan rate $100 \mathrm{mV} \mathrm{s}^{-1}$.

voltammogram showing some peaks, attributed to oxidative process of the carbon film and eventually leading to the destruction of the film. This electrolyte was not used further.

The conclusion was that the potential range which can be used in EIS measurements in acetate buffer solution is between -1.5 and $+1.5 \mathrm{~V}$ (vs SCE).

\subsection{Impedance in the Presence of Dissolved Oxygen}

\subsubsection{Uncoated Carbon Film Disk Electrode}

Impedance spectra recorded in acetate buffer solution after electrode pretreatment are shown in Figure 2. Fitting of spectra was done using an equivalent electrical circuit which comprised the cell resistance, $R_{\Omega}$, in series with a parallel combination of a constant phase element $\mathrm{CPE}_{1}$, as a nonideal capacitor of capacity $C_{1}$ and roughness factor $\alpha_{1}$, where an $\alpha$ value of 1 represents a perfectly smooth surface, in parallel with a resistance, $R_{1}$, representing charge transfer. The CPE was necessary due to the depressed semi-circle character of the responses. This circuit was the same as used in [4] for glassy carbon and in [16] for carbon film cylinder electrodes at frequencies above $1 \mathrm{~Hz}$. Below this frequency, reproducibility between experiments was lower and the form of the spectrum also depended on the applied potential, which made comparisons less easy. The results obtained are shown in Table 1 . The errors in the values in Table 1 were a maximum of $5 \%$ for $R_{1}, 3 \%$ for $C$, and less than $0.5 \%$ for $\alpha$, which is normal for these types of spectra. The cell resistance, $R_{\Omega}$, was unchanged by pretreatment: $9.5 \pm 0.2 \Omega \mathrm{cm}^{2}(N=25)$ and $9.3 \pm 0.3 \Omega \mathrm{cm}^{2}(N=18)$ before and after pretreatment, respectively.

The main conclusions with these disk electrodes are as obtained in [16] at cylinder electrodes. At positive applied potentials, the charge transfer resistance, $R_{1}$, values are higher than at negative potentials and the values increase with pretreatment. However, at negative potentials, they decrease with pretreatment, which could be due to easier
Table 1. Data obtained from analysis of the impedance spectra for carbon film and Nafion-coated carbon film electrodes in the presence of oxygen.

\begin{tabular}{lcccc}
\hline Electrode surface & $\begin{array}{l}E \\
(\mathrm{~V} \text { vs. SCE })\end{array}$ & $\begin{array}{l}R_{1} \\
\left(\mathrm{k} \Omega \mathrm{cm}^{2}\right)\end{array}$ & $\begin{array}{l}C_{1} \\
\left(\mu \mathrm{F} \mathrm{cm} \mathrm{cm}^{-2}\right)\end{array}$ & $\alpha_{1}$ \\
\hline Not pretreated & -1.5 & 2.6 & 8.4 & 0.94 \\
& -1.0 & 10.4 & 14.1 & 0.91 \\
& -0.5 & 7.2 & 19.1 & 0.91 \\
& 0 & 47.9 & 27.7 & 0.88 \\
& 0.5 & 77.6 & 15.2 & 0.91 \\
Pretreated & 1.0 & 54.9 & 10.1 & 0.93 \\
& 1.5 & 9.5 & 5.1 & 0.96 \\
& -1.5 & 4.0 & 11.2 & 0.94 \\
& -1.0 & 6.5 & 13.4 & 0.94 \\
& -0.5 & 3.8 & 18.1 & 0.92 \\
With Nafion & 0 & 20.4 & 31.0 & 0.88 \\
& 0.5 & 123 & 16.3 & 0.93 \\
& 1.0 & 126 & 10.4 & 0.92 \\
& 1.5 & 5.6 & 7.01 & 0.95 \\
& -1.5 & 0.2 & 27.9 & 0.87 \\
& -0.5 & 8.9 & 35.9 & 0.85 \\
& 0 & 3.8 & 46.4 & 0.84 \\
& 0.5 & 9.7 & 58.4 & 0.83 \\
& 1.0 & 28.4 & 38.0 & 0.85 \\
& 1.5 & 29.3 & 29.5 & 0.86 \\
& 2.7 & 15.2 & 0.90 \\
\hline
\end{tabular}

reduction of surface oxide and of dissolved oxygen, particularly evident at $-0.5 \mathrm{~V}$. At the limits of applied potential $(-1.5 \mathrm{~V}$ and $+1.5 \mathrm{~V})$ the lowest values obtained were due to some evolution of hydrogen and oxygen, respectively.

Capacitance values increase with the pretreatment for all the potentials applied to the electrode reflecting the more active surface and higher charge separation efficiency. The $C$ values are also smaller when hydrogen or oxygen evolution occurs. The values of $\alpha_{1}$ are essentially unchanged by pretreatment.

\subsubsection{Nafion-Coated Carbon Film Disk Electrodes}

Figure 2 also shows typical impedance spectra obtained in acetate buffer solution for pretreated carbon film disk electrodes with Nafion coating. The equivalent circuit analysis is given in Table 1 using the same circuit and frequency range as for uncoated electrodes. At more positive potentials, the impedance magnitude is lower with the Nafion coating, but this changes at negative potentials, except at $-1.0 \mathrm{~V}$. At $0.0 \mathrm{~V}$ and at $-1.0 \mathrm{~V}$ the behavior is closer to that of a non-ideal capacitor, but at $-0.5 \mathrm{~V}$ oxygen reduction is clearly occurring. The spectrum obtained at $-1.0 \mathrm{~V}$ (vs. SCE) shows a significant difference in the shape: carbon film electrodes show a semi-circle, and Nafion coated carbon film electrodes give an almost straight line, evidence of charge separation at the Nafion /carbon film substrate interface.

There was a slight increase in cell resistance of ca. $1 \Omega$ $\mathrm{cm}^{-2}$ up to $10.5 \pm 0.4 \Omega \mathrm{cm}^{-2}(N=27)$, due to the Nafion coating.

(c) 2005 WILEY-VCH Verlag GmbH \& Co. KGaA, Weinheim 

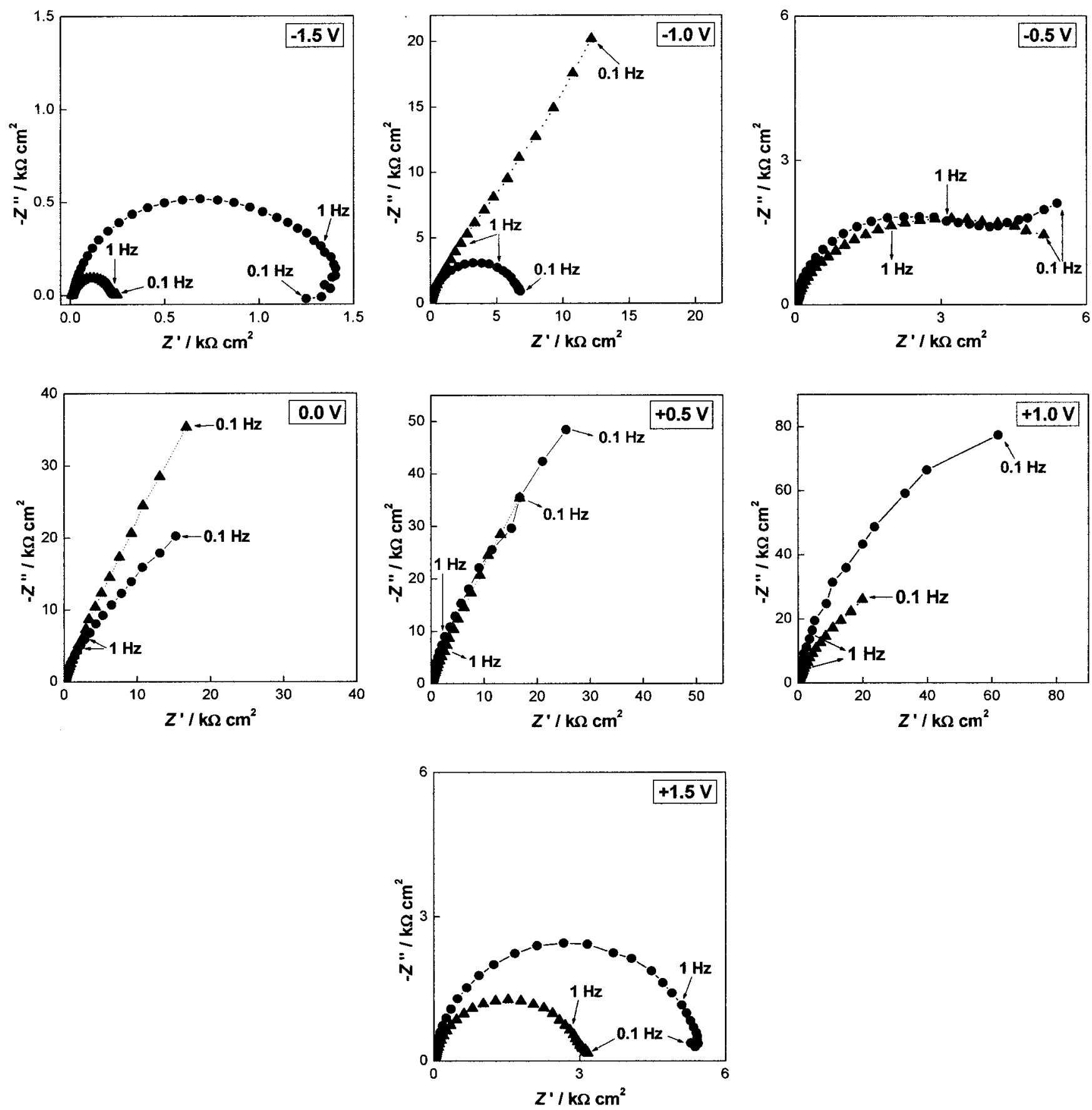

Fig. 2. Complex plane impedance plots for carbon film disk electrodes in $\mathrm{pH} 4.4$ acetate buffer solution: $(\bullet)$ after pretreatment and (ム) with Nafion coating.

As would be predicted with a cation-exchange polymer, there is a significant increase in the capacitance, $C_{1}$. Also, the surface of the Nafion-coated carbon film electrode is rougher than without the coating, corresponding to lower values of $\alpha_{1}$, as seen from Table 1 .

The values of $R_{1}$ increase at negative potentials, which can be due to the limitations imposed to charge transfer by the polymer coating; the same behavior was previously observed in Nafion-coated glassy carbon electrodes [4]. At

Electroanalysis $\mathbf{2 0 0 5}, 17$, No. 7 positive potentials, $R_{1}$ decreases with respect to the bare electrode and charge transfer becomes easier.

It was also found that with the Nafion coating the background current remains stable over time even after impedance measurements at $-1.5 \mathrm{~V}$ or at $+1.5 \mathrm{~V}$. On the other hand, carbon film electrodes without Nafion, before and after pretreatment, need to be conditioned by potential cycling between $-1.5 \mathrm{~V}$ and $+1.5 \mathrm{~V}$ (vs. SCE) to stabilize the background current after recording spectra. 

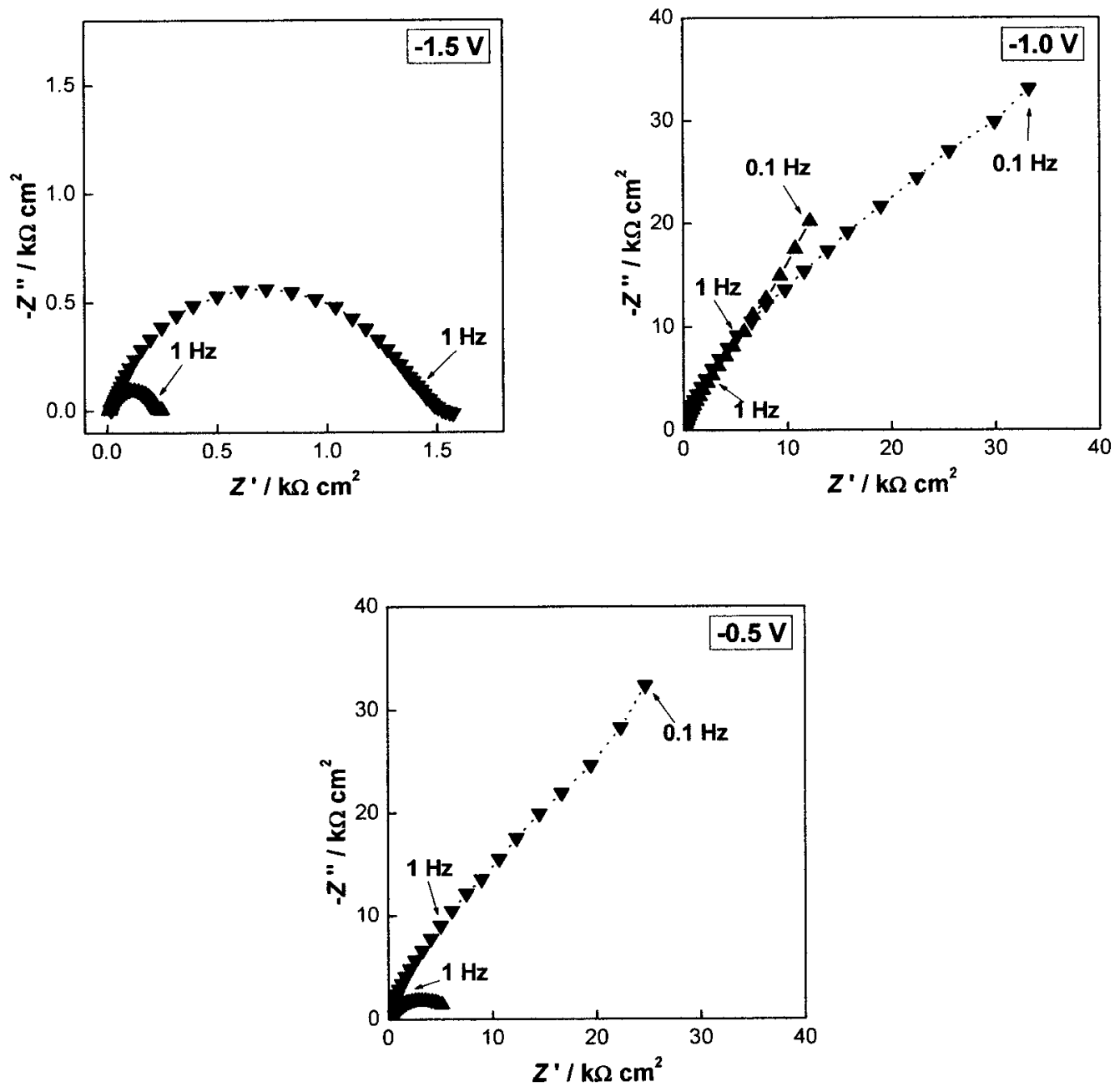

Fig. 3. Complex plane impedance plots for Nafion-coated carbon film disk electrodes in $\mathrm{pH} 4.4$ acetate buffer solution: (ム) with dissolved oxygen and $(\boldsymbol{\nabla})$ without oxygen.

\subsection{Impedance after Removal of Oxygen}

To throw light on the influence of the presence of the oxygen on the electrode reactions, impedance measurements were also made in the absence of oxygen in solution at potentials where oxygen can be reduced, i.e. $-1.5 \mathrm{~V}$, $-1.0 \mathrm{~V}$ and $-0.5 \mathrm{~V}$ (vs. SCE). Figure 3 shows spectra for Nafion-coated carbon film electrodes with and without dissolved oxygen. As can be seen, there is a large increase in the impedance magnitude in solutions without oxygen at $-0.5 \mathrm{~V}$ and at $-1.5 \mathrm{~V}$. This behavior was also observed at glassy carbon electrodes [4] and at $-0.5 \mathrm{~V}$ can be attributed to much more difficult charge transfer processes due to oxygen reduction and being mainly due to surface oxide reduction.

Table 2 summarizes results obtained after fitting the data for uncoated and coated electrodes. The errors obtained on the fitting were similar to those for the values on Table 1.

Comparing the data at negative potentials in Table 1 to those in Table 2 , it can be seen that $R_{1}$ increases dramatically for all the electrodes at $-1.0 \mathrm{~V}$ and $-0.5 \mathrm{~V}$ (vs. SCE) when
Table 2. Data obtained from analysis of the impedance spectra for carbon film and Nafion-coated carbon film electrodes in the absence of oxygen.

\begin{tabular}{llccc}
\hline Electrode surface & $\begin{array}{l}E \\
(\mathrm{~V} \text { vs. SCE })\end{array}$ & $\begin{array}{l}R_{1} \\
\left(\mathrm{k} \Omega \mathrm{cm}^{2}\right)\end{array}$ & $\begin{array}{l}C_{1} \\
\left(\mu \mathrm{F} \mathrm{cm} \mathrm{cm}^{-2}\right)\end{array}$ & $\alpha_{1}$ \\
\hline Not pretreated & -1.5 & 0.5 & 6.2 & 0.98 \\
& -1.0 & 112 & 9.8 & 0.96 \\
& -0.5 & 85.8 & 17.2 & 0.93 \\
Pretreated & -1.5 & 0.4 & 7.2 & 0.97 \\
& -1.0 & 137 & 10.1 & 0.95 \\
With Nafion & -0.5 & 159 & 16.5 & 0.93 \\
& -1.5 & 3.4 & 11.9 & 0.82 \\
& -1.0 & 54.1 & 16.3 & 0.82 \\
& -0.5 & 57.4 & 20.3 & 0.80 \\
\hline
\end{tabular}

oxygen is removed. This increase is accompanied by a decrease in the interfacial capacitance. For electrodes with Nafion there is also a decrease in $\alpha_{1}$, due to its rougher surface, as before.

(C) 2005 WILEY-VCH Verlag GmbH \& Co. KGaA, Weinheim 


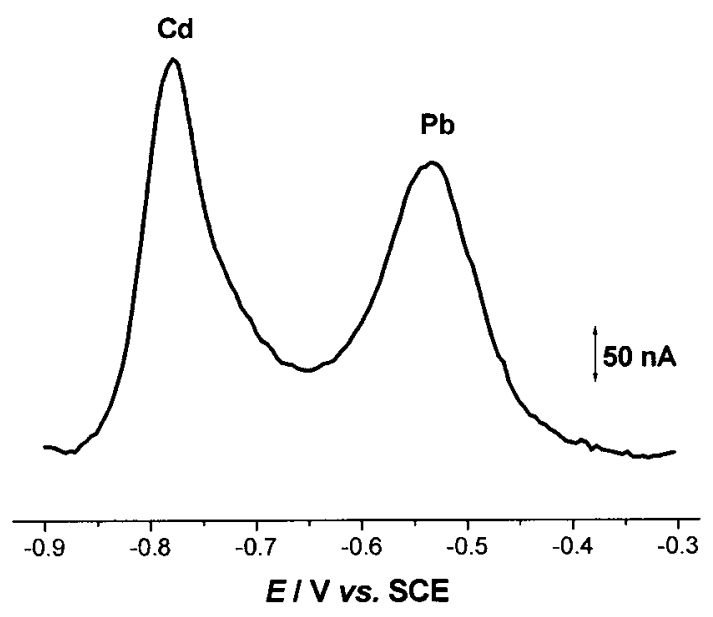

Fig. 4. Square-wave anodic stripping voltammogram of cadmium and lead ions in $0.1 \mathrm{M}$ acetate buffer, $\left[\mathrm{Cd}^{2+}\right]=\left[\mathrm{Pb}^{2+}\right]=10^{-7} \mathrm{M}$. $E_{\mathrm{dep}}=-1.0 \mathrm{~V}$ (vs. SCE), $t_{\mathrm{dep}}=120 \mathrm{~s}$. Square-wave conditions: amplitude $20 \mathrm{mV}$, frequency $25 \mathrm{~Hz}$, potential increment $1 \mathrm{mV}$.

\subsection{Impedance in the Presence of Metal Ions}

The presence of metal ions in solution, in similar concentrations as those used in anodic stripping voltammetry (ASV) experiments, was investigated at Nafion-coated carbon film disk electrodes to probe any reversible or irreversible alterations in electrode behavior.

The experimental square-wave ASV voltammogram of cadmium and lead ions at Nafion-coated carbon film disk electrodes in acetate buffer of Figure 4 demonstrates that they can be determined simultaneously with good peak separation and that the peak potentials are $-0.78 \mathrm{~V}$ and $-0.55 \mathrm{~V}$ (vs. SCE), respectively.

For impedance experiments, $10^{-7} \mathrm{M} \mathrm{Cd}^{2+}$ and $\mathrm{Pb}^{2+}$ ions were added to the acetate buffer solution. A potential of $-1.0 \mathrm{~V}$ (vs. SCE) was then applied for $10 \mathrm{~min}$ in stirred solution, for deposition to occur, and a spectrum was then
Table 3. Data obtained from analysis of the impedance spectra for Nafion-coated carbon film electrodes in $0.1 \mathrm{M}$ acetate buffer pH 4.4 containing $10^{-7} \mathrm{M} \mathrm{Cd}^{2+}$ and $\mathrm{Pb}^{2+}$.

\begin{tabular}{llll}
\hline$E(\mathrm{~V}$ vs. SCE $)$ & $R_{1}\left(\mathrm{k} \Omega \mathrm{cm}^{2}\right)$ & $C_{1}\left(\mu \mathrm{F} \mathrm{cm}^{-2}\right)$ & $\alpha_{1}$ \\
\hline-1.0 & 38.1 & 11.6 & 0.88 \\
-0.5 & 9.0 & 12.4 & 0.90 \\
\hline
\end{tabular}

recorded at $-1.0 \mathrm{~V}$. The electrode was then left at open circuit for $10 \mathrm{~min}$ for re-oxidation to occur and a spectrum recorded at $-0.5 \mathrm{~V}$.

Spectra are shown in Figure 5 and analysis of the results is given in Table 3 using the same equivalent circuit as for the analysis of the previous spectra and over the same frequency range. It can be seen that in the presence of $\mathrm{Cd}^{2+}$ and $\mathrm{Pb}^{2+}$, although the magnitude of the impedance is greater than in their absence, the shape of the spectra is not altered by the presence of metal ions in solution.

At a potential of $-1.0 \mathrm{~V}$, the impedance values are larger after deposition, reflected by a higher charge transfer resistance (presumably owing to partial blocking of the polymer coating near the electrode surface by metal) and a lower capacitance. At $-0.5 \mathrm{~V}$, when the metal ions have been removed, it can also be seen that the charge transfer resistance remains higher than its initial value before metal ion deposition (compare Tables 1 and 2 ) and the capacitance also has been irreversibly decreased. These observations are in agreement with a physical distortion of the Nafion film, caused by the formation of tiny metal deposits within the Nafion pores on the electrode substrate surface. Repeating the process with the same Nafion-coated carbon film electrode, i.e., redeposition and re-oxidation, does not give rise to any further modifications in behavior, so there has been an irreversible change to the structure of the Nafion coating. This suggests that excessive metal deposition may lead to destruction of the coated electrode's ability to give accurate and reproducible results.
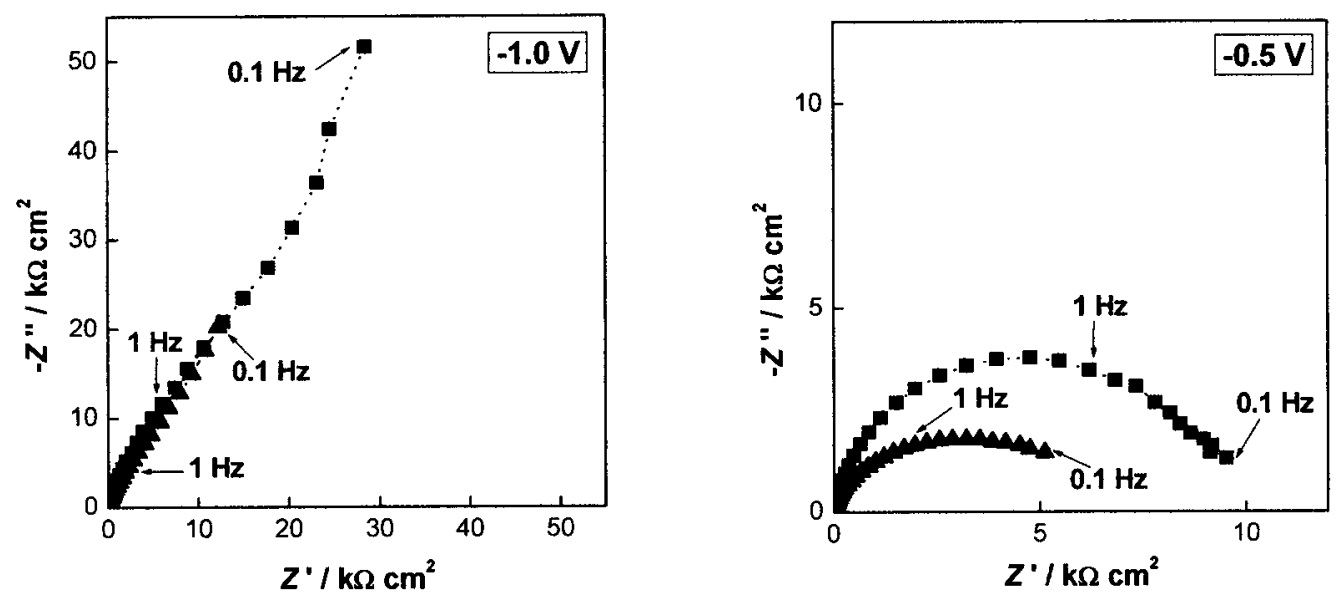

Fig. 5. Complex plane impedance plots for Nafion coated carbon film electrodes in: (ム) pH 4.4 acetate buffer and (๘) in pH 4.4 acetate buffer containing $10^{-7} \mathrm{M} \mathrm{Cd}^{2+}$ and $\mathrm{Pb}^{2+}$. 


\section{Conclusions}

Carbon film disk electrodes for application in anodic stripping voltammetry of metal ions were characterized by EIS. Coating the electrodes with Nafion does not lead to observable changes in the potential window or background current in acetate buffer solution, often used in stripping electroanalysis.

EIS data clearly show evidence of the differences in behavior between Nafion-coated electrodes and uncoated electrodes. Coated electrodes shows a decrease in magnitude of impedance at positive potentials and an increase at negative potentials, with a higher capacitance value and greater roughness, compared with pretreated uncoated carbon film electrodes.

Irreversible changes to the polymer coating of the Nafioncoated carbon film electrodes caused by metal deposition when used in anodic stripping voltammetric analysis for the determination of cadmium and lead ions, were convincingly demonstrated by EIS.

EIS and this type of approach is extremely useful for assessing the upper boundaries for metal deposition within which polymer coatings can be successfully employed without losing their properties in stripping voltammetry on mercury-free solid electrodes for analysis of natural samples.

\section{Acknowledgement}

Financial support from Fundação para a Ciência e Tecnologia (FCT), ICEMS (Research Unit 103), is gratefully acknowledged.

\section{References}

[1] R. L. McCreery, Electroanalytical Chemistry, Vol. 17 (Ed: A. J. Bard), Marcel Dekker, New York 1991.

[2] C. M. A. Brett, Electroanalysis 1999, 11, 1013.

[3] C. M. A. Brett, A. M. Oliveira-Brett, F.-M. Matysik, S. Matysik, S. Kumbhat, Talanta 1996, 43, 2015.

[4] C. M. A. Brett, V. A. Alves, D. A. Fungaro, Electroanalysis 2001, 13, 212.

[5] C. M. A. Brett, D. A. Fungaro, J. M. Morgado, M. H. Gil, J. Electroanal. Chem. 1999, 468, 26.

[6] C. M. A. Brett, D. A. Fungaro, Talanta 2000, 50, 1223.

[7] K. Hayama, H. Tanaka, M. J. Ju, K. Hayashi, K. Toko, Sensors Mater. 2002, 14, 443.

[8] X. Y. Cui, D. C. Martin, Sens. Actuators A 2003, 103, 384.

[9] E. Katz, I. Willner, Electroanalysis 2003, 15, 913.

[10] S. Brahim, A. M. Wilson, D. Narinesingh, E. Iwuoha, A. Guiseppi-Elie, Microchim. Acta 2003, 143, 123.

[11] A. Zeng, E. Liu, S. N. Tan, S. Zhang, J. Gao, Electroanalysis 2002, 14, 1294.

[12] A. Zeng, E. Liu, S. Zhang, S. N. Tan, P. Hing, I. F. Annergren, J. Gao, Thin Solid Films 2003, 426, 258.

[13] A. Zeng, E. Liu, S. Zhang, S. N. Tan, L. X. Liu, J. Gao, Mat. Sci. Forum 2003, 437, 467.

[14] C. M. A. Brett, L. Agnes, H. D. Liess, Electroanalysis 2001, 13,765 .

[15] O. M. S. Filipe, C. M. A. Brett, Talanta 2003, 61, 643.

[16] O. M. S. Filipe, C. M. A. Brett, Electroanalysis 2004, 16, 994. 